\title{
The polypharmacology browser: a web-based multi-fingerprint target prediction tool using ChEMBL bioactivity data
}

Mahendra Awale and Jean-Louis Reymond*

\begin{abstract}
Background: Several web-based tools have been reported recently which predict the possible targets of a small molecule by similarity to compounds of known bioactivity using molecular fingerprints (fps), however predictions in each case rely on similarities computed from only one or two fps. Considering that structural similarity and therefore the predicted targets strongly depend on the method used for comparison, it would be highly desirable to predict targets using a broader set of fps simultaneously.
\end{abstract}

Results: Herein, we present the polypharmacology browser (PPB), a web-based platform which predicts possible targets for small molecules by searching for nearest neighbors using ten different fps describing composition, substructures, molecular shape and pharmacophores. PPB searches through 4613 groups of at least 10 same target annotated bioactive molecules from ChEMBL and returns a list of predicted targets ranked by consensus voting scheme and $p$ value. A validation study across 670 drugs with up to 20 targets showed that combining the predictions from all 10 fps gives the best results, with on average $50 \%$ of the known targets of a drug being correctly predicted with a hit rate of $25 \%$. Furthermore, when profiling a new inhibitor of the calcium channel TRPV 6 against 24 targets taken from a safety screen panel, we observed inhibition in 5 out of 5 targets predicted by PPB and in 7 out of 18 targets not predicted by PPB. The rate of correct (5/12) and incorrect (0/12) predictions for this compound by PPB was comparable to that of other web-based prediction tools.

Conclusion: PPB offers a versatile platform for target prediction based on multi-fingerprint comparisons, and is freely accessible at www.gdb.unibe.ch as a valuable support for drug discovery.

Keywords: Target prediction, Polypharmacology, Drug-target interactions, Molecular fingerprints

\section{Background}

The vast majority of small molecule drugs interact with multiple targets, a general phenomenon known as polypharmacology and a key parameter to be addressed in the course of drug development [1-3]. Many computational tools have been developed that exploit databases containing detailed structural information on the activity of small molecule drugs [4-8] and their protein targets [9] to predict the polypharmacology of any hit compound or drug candidate [10-37]. Several of these tools are

\footnotetext{
*Correspondence: jean-louis.reymond@dcb.unibe.ch Department of Chemistry and Biochemistry, National Center of Competence in Research NCCR Chemical Biology and NCCR TransCure, University of Berne, Freiestrasse 3, 3012 Berne, Switzerland
}

accessible as target prediction websites (Table 1). Each of these websites returns a list of predicted targets based on similarity calculations using molecular fingerprints (fps) or on docking scores.

Ligand-based methods using fp comparisons are particularly versatile because they are applicable to any biological activity, i.e. the target may be a protein but also a cell line or a whole organism. Although some of these ligand based tools offer a selection of different fps, none of them permits the simultaneous use of more than one fp. Considering the fact that molecular similarity and therefore the predicted targets strongly depend on which fp is used for comparison, it would be highly desirable to predict targets using multiple fps simultaneously, 
Table 1 Publicly accessible web-based target prediction tools

\begin{tabular}{|c|c|c|c|}
\hline Website & Similarity method & Database & Ref. \\
\hline www.gdb.unibe.ch & 10 different fingerprints & ChEMBL 21 & This work \\
\hline http://www.pharmaexpert.ru/passonline/ & $\begin{array}{l}\text { Multilevel neighbourhoods of atoms (MNA) } \\
\text { descriptors }\end{array}$ & WDI and ACD & {$[10]$} \\
\hline http://www.dddc.ac.cn/tarfisdock/ & Docking & PDTD & {$[12]$} \\
\hline http://sea.bkslab.org & ECfp4 & CHEMBL 16, WOMBAT, MDDR and StarLite & {$[14]$} \\
\hline http://59.78.96.61/pharmmapper/ & Receptor-based pharmacophore models & TargetBank, DrugBank, BindingDB, PDTD & {$[17]$} \\
\hline https://cpi.bio-x.cn/drar/ & Docking & PDB, DrugBank & {$[18]$} \\
\hline www.cbligand.org/TargetHunter & ECfp6, ECfp4 and Openbabel FP2 & ChEMBL 11 and PubChem bioassay & {$[22]$} \\
\hline http://lilab.ecust.edu.cn/chemmapper/ & Openbabel FP2, MACSS, SHAFT and USR & $\begin{array}{l}\text { ChEMBL 14, BindingDB, DrugBank, KEGG } \\
\text { and PDB }\end{array}$ & {$[23]$} \\
\hline $\begin{array}{l}\text { http://mips.helmholtz-muenchen.de/proj/ } \\
\text { hitpick }\end{array}$ & Circular fingerprint FCFP & STITCH & {$[24]$} \\
\hline http://modlab-cadd.ethz.ch/software/spider/ & CATS and MOE physiochemical descriptors & COBRA & {$[26]$} \\
\hline www.swisstargetprediction.ch & Openbabel FP2 and Electroshape descriptors & ChEMBL 16 & {$[28]$} \\
\hline http://prediction.charite.de/index.php & ECfp4 & ChEMBL, SuperTarget and BindingDB & {$[29]$} \\
\hline www.dddc.ac.cn/tarpred & ECfp4 & BindingDB & {$[33]$} \\
\hline http://potentia.cbs.dtu.dk/ChemProt & Sfp & $\begin{array}{l}\text { ChEMBL 14, BindingDB, DrugBank, } \\
\text { PharmGKB, PubChem bioassay, WOMBAT, } \\
\text { IUPHAR, CTD and STITCH }\end{array}$ & {$[37]$} \\
\hline
\end{tabular}

provided that each $\mathrm{fp}$ would perform well individually in virtual screening and target prediction test cases. Herein we present the polypharmacology browser (PPB), a multifingerprint browser for target prediction which addresses this issue by performing target predictions searches using six different fps and four fused molecular fingerprints (Ffps) (Table 2). Similarities are measured using the cityblock distance because this similarity measure is rapidly computed and therefore well-suited for web-based similarity searches in large databases [38-45]. PPB searches through 2.7 M ligand-target interactions extracted from ChEMBL 21 and generates a list of predicted targets, each linked to the lists of known actives used for the prediction. PPB validation is presented for 670 drugs of known polypharmacology as well as in a predictive application of off-targets for a recently reported inhibitor of transient receptor potential vanilloid 6 (TRPV6) [46]. PPB is freely accessible at www.gdb.unibe.ch and works on computers, tablets and phones.

\section{Methods \\ Dataset}

We analyzed ChEMBL 21 and constructed the target database containing 4613 groups of at least 10 bioactive molecules with documented activity against the same biological target. Briefly, target database was constructed as follows: initially all targets along with their ligands were retrieved from the ChEMBL version 21 .

Table 2 Molecular fingerprints used for target prediction with PPB

\begin{tabular}{|c|c|c|}
\hline Name & Description & Ref. \\
\hline APfp & 21-D atom-pair fingerprint, perceives molecular shape & [43] \\
\hline Xfp & 55-D atom category extended atom-pair fingerprint, perceives pharmacophores & [43] \\
\hline MQN & $\begin{array}{l}\text { 42-D Molecular Quantum Numbers, scalar fingerprint counting atoms, bonds, polarity and ring features, perceives constitution, } \\
\text { topology and molecular shape }\end{array}$ & [38] \\
\hline SMlfp & 34-D scalar fingerprint counting occurrence of characters in SMILES, perceives rings, aromaticity, and polarity & [42] \\
\hline Sfp & 1024-D binary daylight type substructure fingerprint, perceives detailed substructures & [49] \\
\hline ECfp4 & 1024-D binary circular extended connectivity fingerprint, perceives detailed substructures and pharmacophores & {$[50]$} \\
\hline Ffp1 & Fusion fingerprint, Xfp + SMlfp + Sfp & This work \\
\hline Ffp2 & Fusion fingerprint, $\mathrm{Xfp}+\mathrm{MQN}+\mathrm{SMIfp}$ & This work \\
\hline Ffp3 & Fusion fingerprint, Xfp + SMlfp + Sfp + ECfp4 & This work \\
\hline Ffp4 & Fusion fingerprint, Xfp + MQN + SMlfp + Sfp + ECfp4 & This work \\
\hline
\end{tabular}


For each target we retained the compounds having $\mathrm{IC}_{50}$, $\mathrm{EC}_{50}, \mathrm{GI}_{50}, K_{\mathrm{i}}, K_{\mathrm{D}}$, or potency value of $\leq 10 \mu \mathrm{M}$ or percent inhibition of $>50 \%$. All molecules were processed as nonstereo SMILES and ionized at $\mathrm{pH} 7.4$ using an in-house developed Java program utilizing the JChem chemistry library from ChemAxon Pvt. Ltd. Afterwards, duplicate molecules were removed in the context of each target. Finally, targets with at least 10 bioactive compounds were retained in database. In total, these 4613 targets represent 871,673 unique bioactive compounds and $2.7 \mathrm{M}$ ligand-target interactions. Of these targets, $60 \%$ are single protein type, $55 \%$ are human targets, and $45 \%$ have less than 50 bioactive compounds (Fig. 1a-c).

\section{Fingerprints}

For each of the 871,673 selected ChEMBL compounds we computed each of the six fingerprints described in Table 2. In short, APfp, Xfp, MQN and SMIfp were calculated by using an in house developed Java program utilizing various calculator plugins from JChem chemistry library, in particular TopologyAnalyserPlugin to determine shortest topological path for atom-pair, HBDAplugins to determine hydrogen bond donor and acceptor atoms, and MajorMicrospeciesPlugin to adjust the ionization state of molecules. The detailed procedure for the generation of these fingerprints can be found in the respective publication [38, 42, 43]. For Sfp, a daylight type 1024-bit hash fingerprint was computed using the ChemicalFingerprint class of the JChem library. The 1024-bit extended connectivity fingerprint (ECfp4) was calculated with bond diameter of 4 using ECFP class of the JChem library. The source codes for computation of fingerprints are freely available for download at www. gdb.unibe.ch.

\section{Fused fingerprints (Data fusion)}

To generate additional molecular fingerprint descriptions we further investigated data fusion between different combinations of these fingerprints [47]. Since we aimed at using the city-block distance (Eq. 1) as similarity measure, we scaled each fingerprint by analyzing the distance distribution of $50 \mathrm{M}$ random pairs of compounds in each fingerprint space, and scaled values to adjust the most frequently occurring distance in each $\mathrm{fp}$ to the value for Xfp (Fig. 1d/e). We then performed enrichment studies of ligands against decoys in the directory of useful decoys (DUD) [48] and evaluated the average performance of 57 different combinations of the scaled fingerprints in terms of area under the curve (AUC) and enrichment factor at $1 \%$ screening in the receiver operator characteristic (ROC) curves (data not shown). We selected the four fusion fingerprints Ffp1-Ffp4 (Table 2) due to their good performance in this enrichment study (Fig. 1f/g), and computed the corresponding Ffp1-4 values for the 871,673 ChEMBL compounds.

$$
C B D_{A, B}=\sum_{j=1}^{K}\left|A_{j}-B_{j}\right|
$$

\section{$p$ value calculation}

Each target prediction for a given query molecule is based on the city-block distance between the query molecule and the closest member of a group of compounds associated with this target. A $p$ value can be computed for each prediction as the degree of randomness of the observed city block distance [51] and therefore the probability that the corresponding query-target association occurs at random. To compute the $p$ value, we generated a random distance distribution for each of the 4613 targets in each of the ten fingerprint spaces by computing distances between the ChEMBL compounds associated with the target and randomly selected molecules from the ZINC database [52], taken as representative molecules of screening compounds for which target predictions might be carried out (Fig. 1h). For each target up to $1 \mathrm{M}$ random pairs were considered. We then fitted each of the 46,130 distance distributions using a negative binomial distribution function, and generated the corresponding cumulative density functions giving the $p$ value as a function of the city block distance (Fig. 1i). The choice of a negative binomial distribution function was based on the discrete nature of the city-block distance. As the $p$ value calculation is specific for each target protein and fingerprint space it can only be used in this context, and should not be used to compare molecules from different targets or fingerprint spaces. The curve fitting was carried out using the $\mathrm{R}$ statistical package version 3.2.5 using the "fitdistrplus" library with default maximum likelihood method for parameters estimation.

\section{Validation set}

The validation set containing 670 drugs and their targets annotation was created as follows: (a) compounds labelled as approved drug or drug in clinical trial were extracted from ChEMBL database, (b) for each drug, target list was constructed by comparing SMILES string of drug to SMILES of bioactive compounds of targets used in the PPB. When the SMILES string was matched, corrosponding PPB target was added to known target list for the drug and (c) retained the drugs with $\leq 20$ targets in the list.

\section{The PPB web-interface}

Given a query molecule, PPB computes each of the 10 fps for this compound, sorts the 871,673 ChEMBL compounds by city-block distance to the query using each $\mathrm{fp}$ 


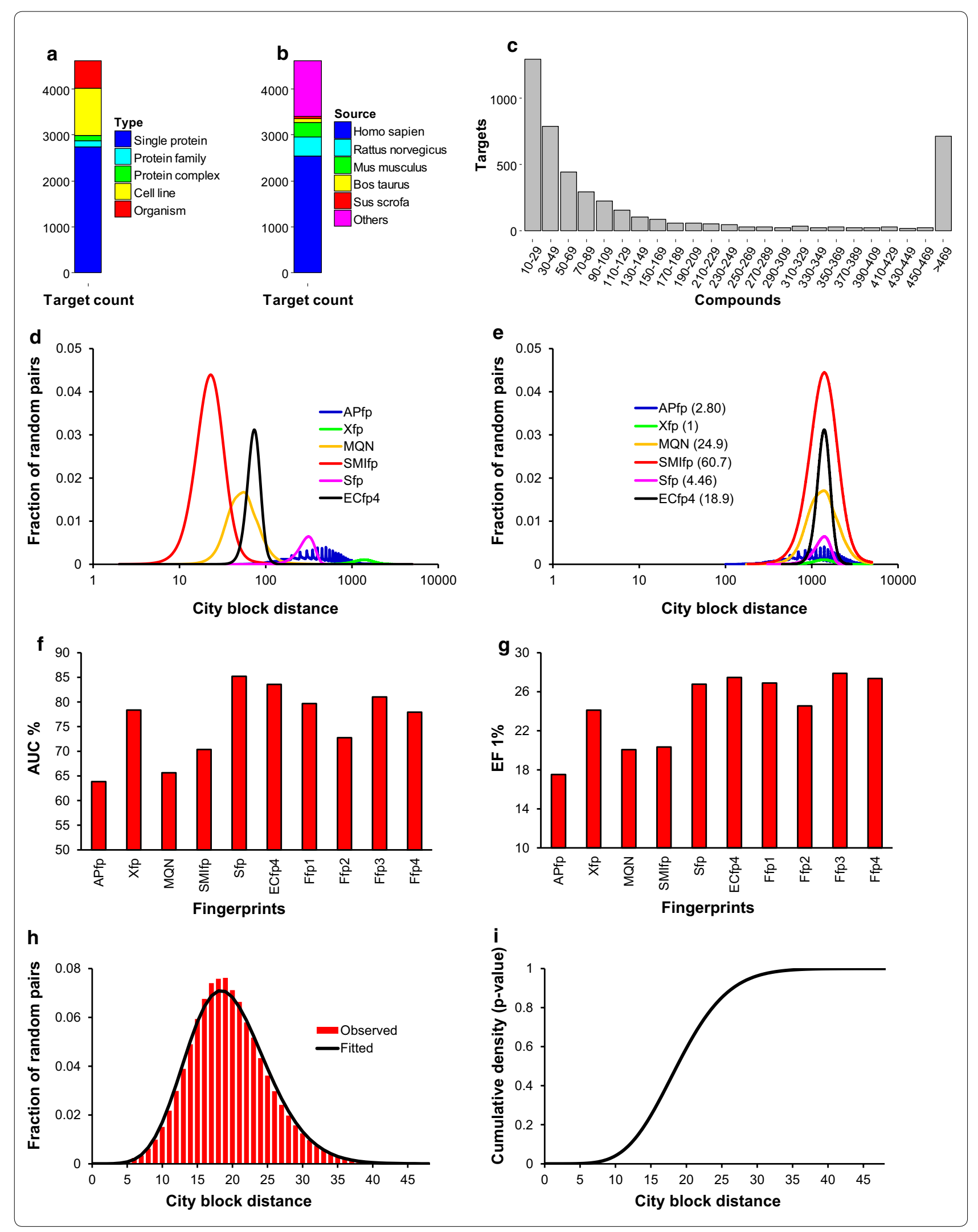


(See figure on previous page.)

Fig. 1 Overview of the data used for constructing PPB. Distribution of a target type as defined in ChEMBL and $\mathbf{b}$ source of targets. c Distribution of targets as per number of associated bioactive compounds. $\mathbf{d}$ Histogram of city block distances (log scale) calculated for 50 million random pairs of compounds from ChEMBL 21 using six molecular fingerprints. e APfp, MQN, SMlfp, Sfp and ECfp4 fingerprints were scaled with respect to Xfp to adjust to the value of the most frequent distance. Scaling factors are shown in parentheses. $\mathbf{f}, \mathbf{g}$ Enrichment of 40 set of DUD actives from corresponding decoys set by six different fingerprints (APfp, Xfp, MQN, SMlfp, Sfp and ECfp4) and four similarity fusion methods (Ffp 1-4). City block distance was used as sorting function. Data is represented as average of $\mathbf{f}$ Area under ROC curve and $\mathbf{g}$ Enrichment factor at $1 \%$ of screen database for 40 targets from DUD. $\mathbf{h}, \mathbf{i}$ Example of $p$ value calculation. $\mathbf{h}$ Observed (red) and fitted (black) random distance distributions for the muscarinic acetylcholine receptor M1 (CHRM1, CHEMBL216) in MQN fingerprint space. City block distances were calculated for 1788 ligands of CHRM1 with respect to random compounds from ZINC database. Negative binomial distribution was used for curve fitting. i Cumulative density plot indicating area under fitted curve in $\mathbf{h}$

independently, and collects a predefined number of compound associated targets in each case. The $p$ value for each target is calculated from the nearest neighbor found for that target. Finally, PPB merges the different target lists.

The graphical user interface of PPB starts with an initial page wherein the user can input the structure of a query molecule using the JavaScript based JSME molecular editor (http://peter-ertl.com/jsme/) [53]. The structure can be drawn or copy-pasted in SMILES or sdf file format. An option is available to extract the query molecule from the Protein Data Bank (PDB) using the PDB id of the protein-ligand complex of interest (the PDB ligand data was downloaded from http:/ligand-expo.rcsb.org/ website in March 2016 and stored on our web server, and will be updated once a year). The option "No. of targets" allows the user to input the number of targets to be returned by each fp. By default this parameter is set to 20. Following the entry of a query molecule, the target search can be initiated by clicking on the "Submit" button. Typically, execution of a search takes less than $1 \mathrm{~min}$.

The target prediction results are presented as list of targets annotated with a probability bar for each fp (Fig. 2a). The $p$ value is shown by a green bar of decreasing length up to $p=0.01$, with values above 0.01 written as number in

\section{a}

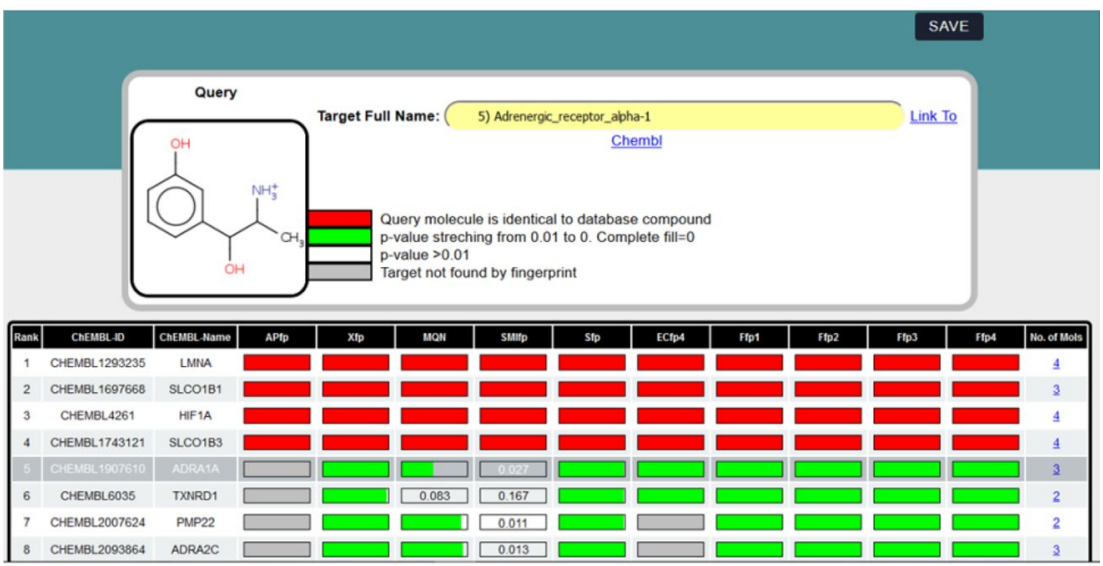

b

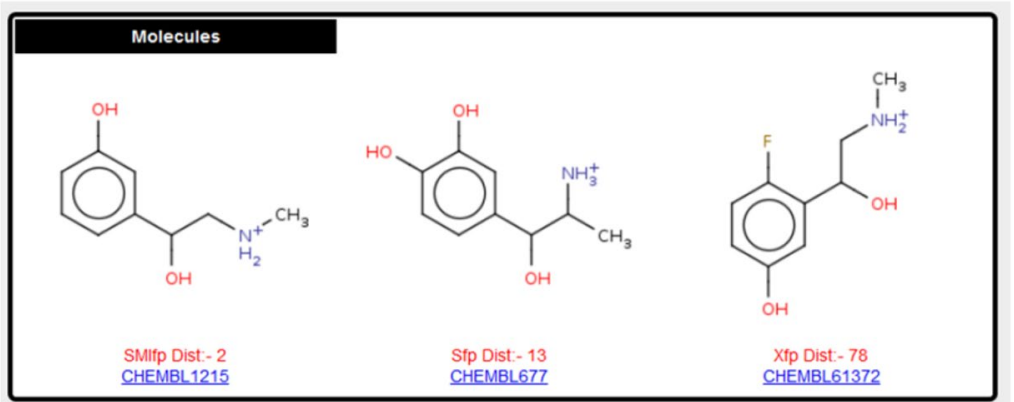

Fig. 2 PPB web-browser. a Result panel displaying the PPB predicted targets for the drug metaraminol. b List of molecules for the target selected in the result panel (row 5 ADRA1A) 
the white probability bar. A grey bar indicates that the target was not found, and a red bar indicates that a molecule with distance $=0$ (usually the identical molecule) is present in the ChEMBL reference list. Each target is labelled with its short name and ChEMBL target id, and the number of compounds retrieved by the browser is indicated in the last column of the table. In the initial display the list of targets is sorted by frequency of occurrence and sum of $p$ values across the $10 \mathrm{fps}$, which defines the target rank (first column). The list can also be sorted by ChEMBL target id, target name, and by the number of selected compounds by clicking on the corresponding column heading.

Clicking on the number of selected compounds per target (furthest right column) opens a new tab displaying the structures of these compounds labelled with fingerprints, city-block distance and ChEMBL compound id (Fig. 2b). The selection of a row in the table displays the full name of the target in the "Target name" field, which is an active link to the parent ChEMBL database to obtain further information on this target. The result (targets and molecules) can be stored as text file using the "save" button provided in each window.

\section{Results and discussion}

The performance of PPB was evaluated by challenging its ability to recall the known targets of 670 compounds labelled as approved drug or drug in clinical trial and annotated with up to 20 targets in ChEMBL (4794 drug-target interactions). Prior to evaluation these 670 drugs were removed from our target compound database. Among these drugs $71 \%$ had less than 10 associated targets and the remaining 29\% had 10-20 associated targets (Fig. 3).

The 670 different searches were performed using PPB with the default search settings. The predicted targets were analysed for each fp considering (a) all targets, (b) targets with $p$ value $\leq 0.01$ and (c) targets with $p$ value

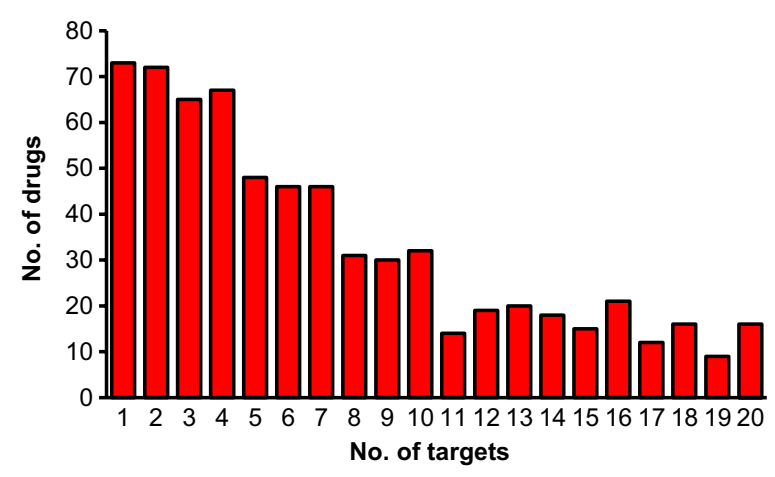

Fig. 3 Distribution of 670 ChEMBL compounds labelled as drug in clinical trial or as approved drug used in validation study according to number of associated targets
$>0.01$ (Fig. 4; Additional file 1: Fig. S1). When combining the results of the different fps (last column, "comb" in Fig. 4a-d) we only considered the targets voted by at least 2 different fps. For each drug, we calculated the fraction of known targets in the results list, the total number of predicted targets and the hit rate (ratio of known targets found to predicted targets).

In terms of the fraction of correctly predicted known targets (Fig. 4a) the fusion fingerprints Ffp1, Ffp3 and Ffp4 showed the highest average value, followed by the binary fingerprints Sfp and ECfp4, the pharmacophore fingerprint Xfp, and finally MQN, SMIfp and APfp. To evaluate if the performance of these fingerprints significantly differ from one another or not, the student $t$ tests (at confidence interval of 0.95) were performed for all the possible pairs of fingerprints (Additional file 1: Tables S1 and S2). Although most of the individual fingerprint pairs show significant differences in performance, no significant differences were found between performance of Sfp, ECfp4, Ffp1 and Ffp2. Overall the performance trend followed the complexity of each fingerprint and highlights that detailed structural encoding tends to increase prediction performance.

These single fp approaches were outperformed by using the combination of all 10 results lists (combined method), however at the expense of a relatively low hit rate resulting from checking a larger number of targets for each drug as compared to individual fps (Fig. 4b/c). The combined method also showed the highest success rate for finding at least one known target among the 5 top predicted targets for each fp (Fig. 4d). For all methods except SMIfp the predicted target list with $p$ value $\leq 0.01$ showed a significantly greater chance of success compared to the target lists with $p$ value $>0.01$. The importance of a low $p$ value for target prediction was particularly striking with the fused fingerprints Ffp1-4 and the combined method, for which more than $75 \%$ of all correct predictions originated from predicted targets with $p$ value $\leq 0.01$.

Although several of the fps were not statistically different in terms of performance, the pairwise overlap between predicted targets by each of the 10 different fps showed that on average less than $45 \%$ of targets were common between any two fps (Fig. 4f). Furthermore each fp retrieved a significant number of unique targets which are not found by any other $\mathrm{fp}$, further highlighting the utility of each fp. Interestingly, APfp and Xfp, which perceive the shape and pharmacophore patterns in molecules, returned the highest percentages of unique targets (52 and $31 \%$ respectively). For fused fingerprints Ffp1-4 the percentages of unique targets were relatively low (3-10\%) due to the considerable overlap among themselves.

A similar analysis performed by categorizing targets according to their $p$ values (Fig. $4 \mathrm{~g} / \mathrm{h}$ ) showed that the 


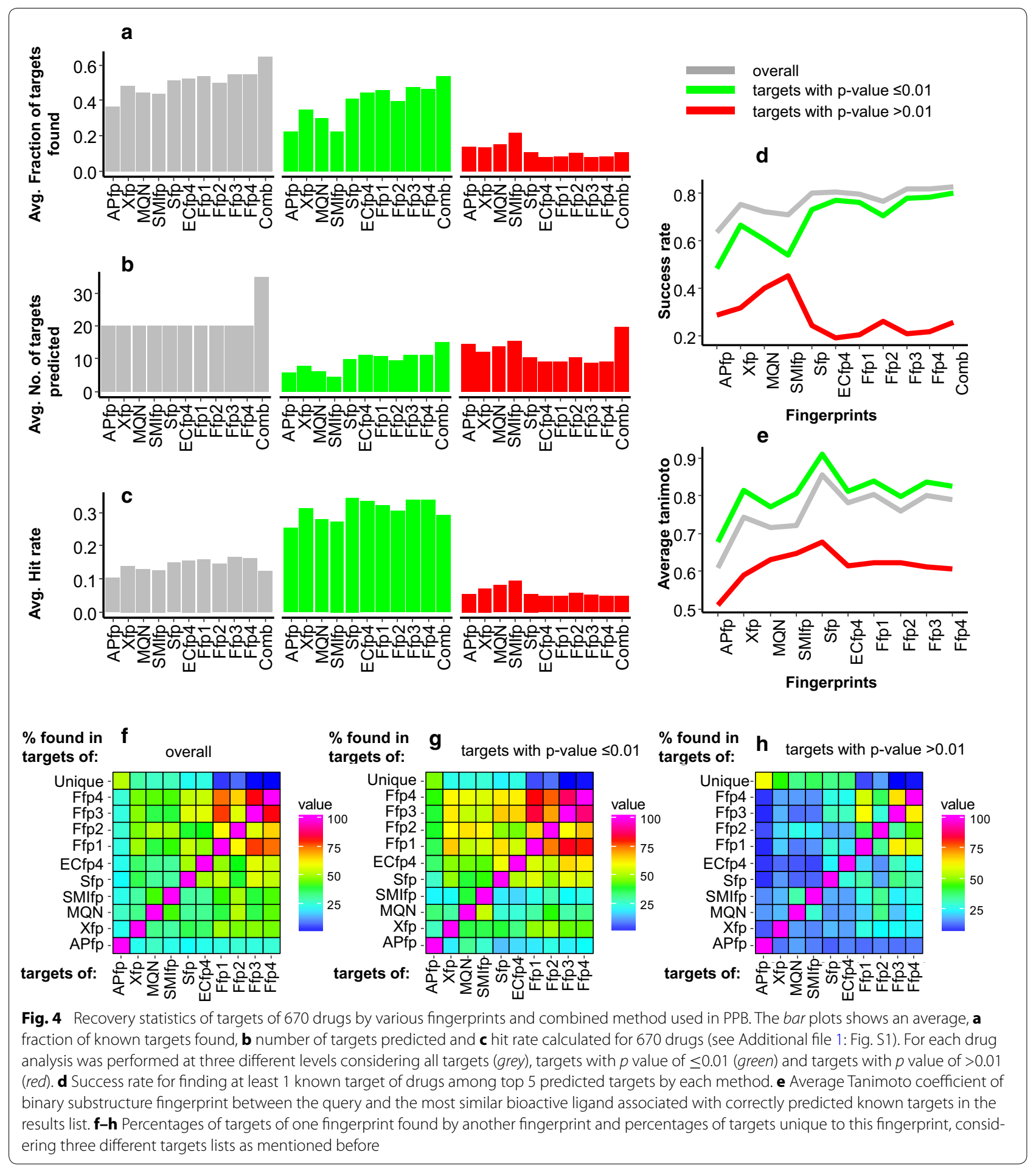

pairwise overlap between high confidence (low $p$ value) targets of different fingerprints were significantly higher (on average $47 \%$ overlap) as compared to low confidence targets (high $p$ value, on average $24 \%$ overlap). This can be explained by the fact that at high $p$ values the structural similarity between a query and its nearest neighbour compound associated with the target becomes less obvious and difficult to capture (Fig. 4e).

\section{Prediction of off-targets of a new TRPV6 inhibitor}

We recently reported the identification of CIS22a (Fig. 5a) as the first potent and selective inhibitor of 


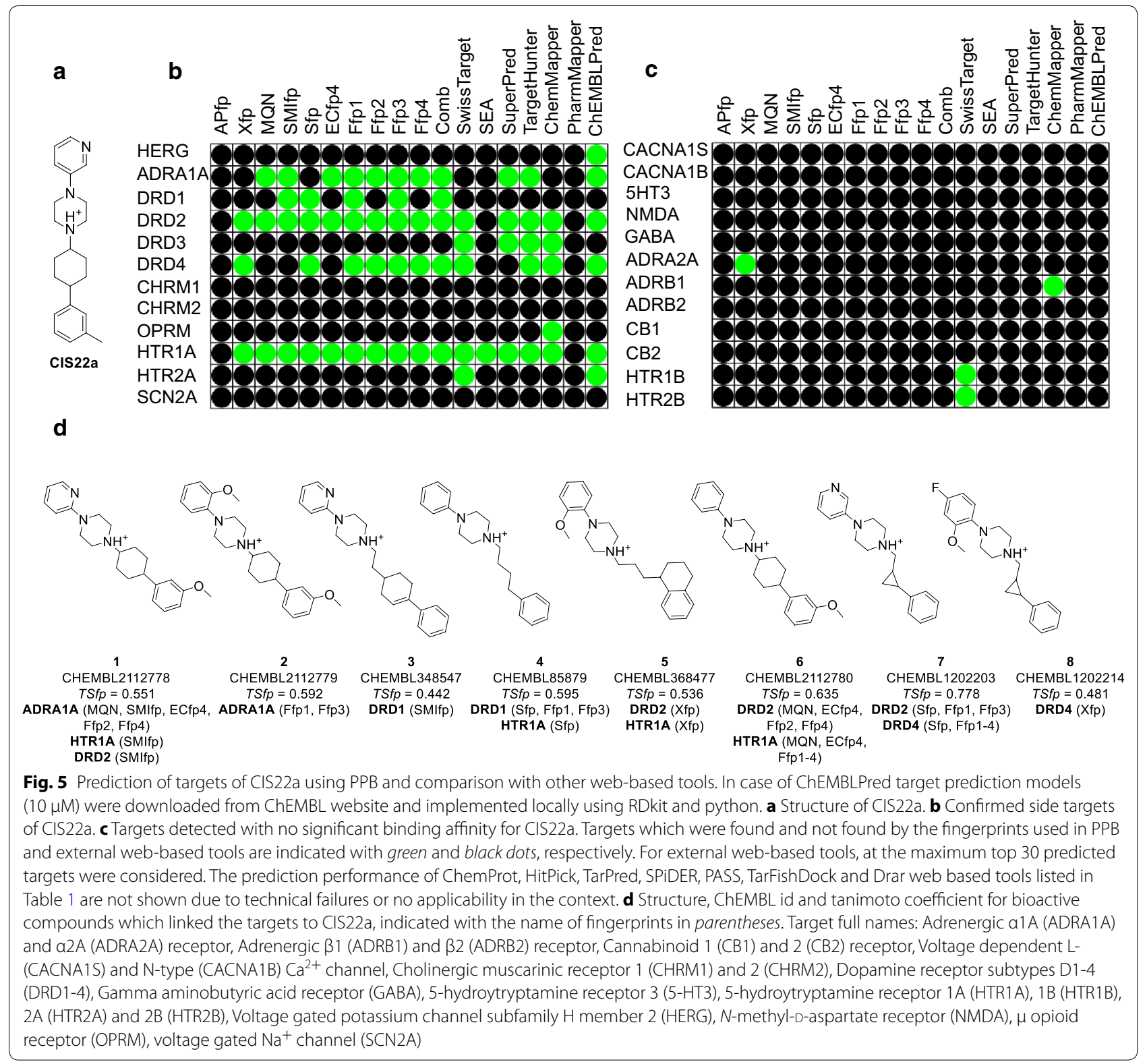

TRPV6, a transmembrane calcium channel overexpressed in breast and prostate cancer [46]. We tested the polypharmacology of this inhibitor for 24 out of 44 targets present in the "safety screen" panel of Cerep Pvt. Ltd (Fig. 5b/c). To assemble this target list we first inspected the PPB results list and chose five targets selected by multiple fps considering in each case the target from human and rat origin as the same target. These were the adrenergic $\alpha 1 \mathrm{~A}$ receptor (ADRA1A), the dopamine receptor subtypes D1 (DRD1), D2 (DRD2), and D4 (DRD4), the 5-hydroxytryptamine receptor 1A (HTR1A). We then added further subtypes of these five targets as well as all ion channels present in the safety screen panel, resulting in a list of 17 GPCRs and seven ions channels.

In-vitro profiling showed that CIS22a bound significantly ( $>50 \%$ inhibition at $10 \mu \mathrm{M}$ ) to 12 targets of the 24 selected targets (Fig. 5b). Five of these targets (ADR1A, DRD1, DRD2, DRD4 and 5HTR1A) were proposed by multiple fps in the PPB. Only two of the Ffps (Ffp1 and Ffp3) and the combined method were able to predict all of the five targets, illustrating the usefulness of similarity fusion methods and combined data analysis. The analysis of bioactive compounds which linked these five targets to CIS22a showed that the linking compounds 
were shared by different fps and closely related targets (1-8 in Fig. 5d). Interestingly, the linking compounds for three targets (DRD2, DRD4 and HTR1A) suggested by Xfp were not shared by any other fps, probably because of their relatively low substructure similarity to the query compound. On the other hand Xfp predicted an activity on ADRA2A, which was not confirmed experimentally (only $31 \%$ inhibition at $10 \mu \mathrm{M}$ ).

For comparison we successfully ran target predictions for CIS22a using six of the fourteen target prediction web-based tools listed in Table 1. Results comparable to PPB were obtained with SwissTarget, SuperPred, TargetHunter, ChemMapper and ChEMBLPred. On the other hand, SEA only returned a single, correct target, and PharmMapper did not predict any of the tested targets.

\section{Conclusion}

The PPB web tool features a unique, intuitive and exhaustive search platform for target prediction. The comparative view of target list from various fingerprint spaces provides a simple yet efficient way for selection of targets by consensus voting. PPB provides a valuable support to drug discovery projects.

\section{Additional file}

Additional file 1. Figure S1: Recovery statistics of targets of 670 drugs by various fingerprints and combined method used in PPB. Tables S1 and S2: Student t-test significance values for pair wise comparison of target prediction performance of all the fingerprints used in PPB.

\section{Authors' contributions}

MA designed and realized the project and wrote the paper. J-LR co-designed and supervised the project and wrote the paper. Both authors read and approved the final manuscript.

\section{Competing interests}

The authors declare that they have no competing interests.

\section{Availability of data and materials}

The website, source code and all the data used for development and validation of PPB is freely available for download at the PPB home page.

\section{Funding}

This work was supported financially by the University of Berne, the Swiss National Science Foundation and the NCCR TransCure.

Received: 29 August 2016 Accepted: 10 February 2017

Published online: 21 February 2017

\section{References}

1. Overington JP, Al-Lazikani B, Hopkins AL (2006) How many drug targets are there? Nat Rev Drug Discov 5(12):993-996

2. Anighoro A, Bajorath J, Rastelli G (2014) Polypharmacology: challenges and opportunities in drug discovery. J Med Chem 57(19):7874-7887
3. Lavecchia A, Cerchia C (2016) In silico methods to address polypharmacology: current status, applications and future perspectives. Drug Discov Today 21(2):288-298

4. Wishart DS, Knox C, Guo AC, Shrivastava S, Hassanali M, Stothard P, Chang Z, Woolsey J (2006) DrugBank: a comprehensive resource for in silico drug discovery and exploration. Nucleic Acids Res 34(suppl 1):D668-D672

5. Olah M, Rad R, Ostopovici L, Bora A, Hadaruga N, Hadaruga D, Moldovan R, Fulias A, Mractc M, Oprea TI (2008) WOMBAT and WOMBAT-PK: bioactivity databases for lead and drug discovery, chemical biology: from small molecules to systems biology and drug design. Wiley-VCH Verlag GmbH, Weinheim, pp 760-786

6. Wang Y, Xiao J, Suzek TO, Zhang J, Wang J, Bryant SH (2009) PubChem: a public information system for analyzing bioactivities of small molecules. Nucleic Acids Res 37(Web Server issue):W623-W633

7. Gaulton A, Bellis LJ, Bento AP, Chambers J, Davies M, Hersey A, Light Y, McGlinchey S, Michalovich D, Al-Lazikani B et al (2012) ChEMBL: a large-scale bioactivity database for drug discovery. Nucleic Acids Res 40(D1):D1100-D1107

8. Gilson MK, Liu T, Baitaluk M, Nicola G, Hwang L, Chong J (2015) BindingDB in 2015: a public database for medicinal chemistry, computational chemistry and systems pharmacology. Nucleic Acids Res 44(D1):D1045-D1053

9. Rose PW, Prlić A, Bi C, Bluhm WF, Christie CH, Dutta S, Green RK, Goodsell DS, Westbrook JD, Woo J et al (2015) The RCSB Protein Data Bank: views of structural biology for basic and applied research and education. Nucleic Acids Res 43(D1):D345-D356

10. Lagunin A, Stepanchikova A, Filimonov D, Poroikov V (2000) PASS: prediction of activity spectra for biologically active substances. Bioinformatics 16(8):747-748

11. Jenkins JL, Bender A, Davies JW (2006) In silico target fishing: predicting biological targets from chemical structure. Drug Discov Today Technol 3(4):413-421

12. Li H, Gao Z, Kang L, Zhang H, Yang K, Yu K, Luo X, Zhu W, Chen K, Shen J et al (2006) TarFisDock: a web server for identifying drug targets with docking approach. Nucleic Acids Res 34(suppl 2):W219-W224

13. Nidhi, Glick M, Davies JW, Jenkins JL (2006) Prediction of biological targets for compounds using multiple-category Bayesian models trained on chemogenomics databases. J Chem Inf Model 46(3):1124-1133

14. Keiser MJ, Roth BL, Armbruster BN, Ernsberger P, Irwin JJ, Shoichet BK (2007) Relating protein pharmacology by ligand chemistry. Nat Biotechnol 25(2):197-206

15. Nigsch F, Bender A, Jenkins JL, Mitchell JBO (2008) Ligand-target prediction using winnow and naive Bayesian algorithms and the implications of overall performance statistics. J Chem Inf Model 48(12):2313-2325

16. Wale N, Karypis G (2009) Target fishing for chemical compounds using target-ligand activity data and ranking based methods. J Chem Inf Model 49(10):2190-2201

17. Liu X, Ouyang S, Yu B, Liu Y, Huang K, Gong J, Zheng S, Li Z, Li H, Jiang $H$ (2010) PharmMapper server: a web server for potential drug target identification using pharmacophore mapping approach. Nucleic Acids Res 38(suppl 2):W609-W614

18. Luo H, Chen J, Shi L, Mikailov M, Zhu H, Wang K, He L, Yang L (2011) DRAR-CPI: a server for identifying drug repositioning potential and adverse drug reactions via the chemical-protein interactome. Nucleic Acids Res 39(Suppl 2):W492-W498

19. Koutsoukas A, Simms B, Kirchmair J, Bond PJ, Whitmore AV, Zimmer S, Young MP, Jenkins JL, Glick M, Glen RC et al (2011) From in silico target prediction to multi-target drug design: current databases, methods and applications. J Proteom 74(12):2554-2574

20. AbdulHameed MDM, Chaudhury S, Singh N, Sun H, Wallqvist A, Tawa GJ (2012) Exploring polypharmacology using a ROCS-based target fishing approach. J Chem Inf Model 52(2):492-505

21. Mavridis L, Mitchell JB (2013) Predicting the protein targets for athletic performance-enhancing substances. J Cheminform 5(1):1-13

22. Wang L, Ma C, Wipf P, Liu H, Su W, Xie X-Q (2013) TargetHunter: an in silico target identification tool for predicting therapeutic potential of small organic molecules based on chemogenomic database. AAPS J 15(2):395-406

23. Gong J, Cai C, Liu X, Ku X, Jiang H, Gao D, Li H (2013) ChemMapper: a versatile web server for exploring pharmacology and chemical structure association based on molecular 3D similarity method. Bioinformatics 29(14):1827-1829 
24. Liu X, Vogt I, Haque T, Campillos M (2013) HitPick: a web server for hit identification and target prediction of chemical screenings. Bioinformatics 29(15):1910-1912

25. Peragovics Á, Simon Z, Tombor L, Jelinek B, Hári P, Czobor P, MálnásiCsizmadia A (2013) Virtual affinity fingerprints for target fishing: a new application of drug profile matching. J Chem Inf Model 53(1):103-113

26. Reker D, Rodrigues T, Schneider P, Schneider G (2014) Identifying the macromolecular targets of de novo-designed chemical entities through self-organizing map consensus. Proc Natl Acad Sci 111(11):4067-4072

27. Clark AM, Sarker M, Ekins S (2014) New target prediction and visualization tools incorporating open source molecular fingerprints for TB Mobile 2.0. J Cheminform 6(1):1-17

28. Gfeller D, Grosdidier A, Wirth M, Daina A, Michielin O, Zoete V (2014) SwissTargetPrediction: a web server for target prediction of bioactive small molecules. Nucleic Acids Res 42(W1):W32-W38

29. Nickel J, Gohlke B-O, Erehman J, Banerjee P, Rong WW, Goede A, Dunkel M, Preissner R (2014) SuperPred: update on drug classification and target prediction. Nucleic Acids Res 42(W1):W26-W31

30. Cereto-Massagué A, Ojeda MJ, Valls C, Mulero M, Pujadas G, Garcia-Vallve $S$ (2015) Tools for in silico target fishing. Methods 71:98-103

31. Lusci A, Fooshee D, Browning M, Swamidass J, Baldi P (2015) Accurate and efficient target prediction using a potency-sensitive influencerelevance voter. J Cheminform $7(1): 1-13$

32. Afzal AM, Mussa HY, Turner RE, Bender A, Glen RC (2015) A multi-label approach to target prediction taking ligand promiscuity into account. J Cheminform 7(1):1-14

33. Liu X, Gao Y, Peng J, Xu Y, Wang Y, Zhou N, Xing J, Luo X, Jiang H, Zheng M (2015) TarPred: a web application for predicting therapeutic and side effect targets of chemical compounds. Bioinformatics 31(12):2049-2051

34. Cao R, Wang Y (2016) Predicting molecular targets for small-molecule drugs with a ligand-based interaction fingerprint approach. ChemMedChem 11(12):1352-1361

35. Wang Z, Liang L, Yin Z, Lin J (2016) Improving chemical similarity ensemble approach in target prediction. J Cheminform 8(1):1-10

36. Wang X, Pan C, Gong J, Liu X, Li H (2016) Enhancing the enrichment of pharmacophore-based target prediction for the polypharmacological profiles of drugs. J Chem Inf Model 56(6):1175-1183

37. Kringelum J, Kjaerulff SK, Brunak S, Lund O, Oprea TI, Taboureau O. ChemProt-3.0: a global chemical biology diseases mapping. Database 2016; 2016.

38. Nguyen KT, Blum LC, van Deursen R, Reymond J-L (2009) Classification of organic molecules by molecular quantum numbers. ChemMedChem 4(11):1803-1805
39. Blum LC, Reymond J-L (2009) 970 million druglike small molecules for virtual screening in the chemical universe database GDB-13. J Am Chem Soc 131(25):8732-8733

40. Blum LC, van Deursen R, Bertrand S, Mayer M, Bürgi JJ, Bertrand D, Reymond J-L (2011) Discovery of a7-nicotinic receptor ligands by virtual screening of the chemical universe database GDB-13. J Chem Inf Model 51(12):3105-3112

41. Ruddigkeit L, Blum LC, Reymond J-L (2013) Visualization and virtual screening of the chemical universe database GDB-17. J Chem Inf Model 53(1):56-65

42. Schwartz J, Awale M, Reymond J-L (2013) SMIfp (SMILES fingerprint) chemical space for virtual screening and visualization of large databases of organic molecules. J Chem Inf Model 53(8):1979-1989

43. Awale M, Reymond J-L (2014) Atom pair 2D-fingerprints perceive 3D-molecular shape and pharmacophores for very fast virtual screening of ZINC and GDB-17. J Chem Inf Model 54(7):1892-1907

44. Awale M, Reymond J-L (2014) A multi-fingerprint browser for the ZINC database. Nucleic Acids Res 42:W234-W239

45. Reymond JL (2015) The chemical space project. Acc Chem Res 48(3):722-730

46. Simonin C, Awale M, Brand M, van Deursen R, Schwartz J, Fine M, Kovacs G, Häfliger P, Gyimesi G, Sithampari A et al (2015) Optimization of TRPV6 calcium channel inhibitors using a 3D ligand-based virtual screening method. Angew Chem Int Ed 54(49):14748-14752

47. Willett $P$ (2013) Fusing similarity rankings in ligand-based virtual screening. Comput Struct Biotechnol J 5(6):1-6

48. Huang N, Shoichet BK, Irwin JJ (2006) Benchmarking sets for molecular docking. J Med Chem 49(23):6789-6801

49. Hagadone TR (1992) Molecular substructure similarity searching: efficient retrieval in two-dimensional structure databases. J Chem Inf Comput Sci 32(5):515-521

50. Rogers D, Hahn M (2010) Extended-connectivity fingerprints. J Chem Inf Model 50(5):742-754

51. Baldi P, Nasr R (2010) When is chemical similarity significant? The statistical distribution of chemical similarity scores and its extreme values. J Chem Inf Model 50(7):1205-1222

52. Irwin JJ, Sterling T, Mysinger MM, Bolstad ES, Coleman RG (2012) ZINC: a free tool to discover chemistry for biology. J Chem Inf Model 52(7):1757-1768

53. Bienfait B, Ertl P (2013) JSME: a free molecule editor in JavaScript. ¡J Cheminform 5(1):1-6

\section{Submit your manuscript to a SpringerOpen ${ }^{\odot}$ journal and benefit from:}

- Convenient online submission

- Rigorous peer review

- Immediate publication on acceptance

- Open access: articles freely available online

- High visibility within the field

- Retaining the copyright to your article

Submit your next manuscript at springeropen.com 